\title{
Report summary - Health-Adjusted Life Expectancy in Canada: 2012 Report by the Public Health Agency of Canada
}

The Public Health Agency of Canada Steering Committee on Health-Adjusted Life Expectancy (1)

Health-adjusted life expectancy is an indicator of the average number of years that an individual is expected to live in a healthy state. It is a summary measure that combines both quantity of life and quality of life. In other words, it combines mortality and morbidity experience into a single summary measure of population health. It can be used to measure the burden of disease and injury in the population, risk factors and the performance of public health efforts.

This report, entitled Health-Adjusted Life Expectancy in Canada: 2012 Report by the Public Health Agency of Canada, ${ }^{1}$ provides estimates of health-adjusted life expectancy among Canadians with and without selected chronic diseases (diabetes and cancer) and chronic conditions (hypertension), and by socio-economic status (income). Estimates are provided for females and males and for people of different ages.

Low socio-economic status is associated with a loss in health-adjusted life expectancy. In 2001, Canadian women and men in the top one-third income group had a health-adjusted life expectancy at birth of 72.3 years and 70.5 years, respectively. Compared with being in the highest income group, being in the bottom onethird income group was associated with a loss of health-adjusted life expectancy at birth of 3.2 years for women and 4.7 years for men.

Chronic diseases and conditions also are associated with a significant loss in healthadjusted life expectancy. The estimates of health-adjusted life expectancy by chronic disease status in this report were calculated based on the mortality and morbidity experience of people with and without diabetes and/or hypertension (high blood pressure) for the 2004-2006 period and of people with and without cancer for the 2002-2005 period. According to the results of this study, the diabetes cohort at age 55 had a loss in health-adjusted life expectancy of 5.8 years for women and 5.3 years for men. The cohort of people with high blood pressure at age 55 had a loss of 2.0 years and 2.7 years for females and males, respectively. The cancer cohort at age 65 had a loss in health-adjusted life expectancy of 10.3 years for women and 9.2 years for men.

This report provides information for use in public health research, policy development and practice. Future reports could extend the scope to include healthadjusted life expectancy by behavioural risk factor status (such as obesity, physical inactivity and smoking).

The full report is available at http://www. phac-aspc.gc.ca/cd-mc/hale-evas-pdf-eng.php

\section{References}

1. Public Health Agency of Canada Steering Committee on Health-Adjusted Life Expectancy. Health-Adjusted Life Expectancy in Canada: 2012 Report by the Public Health Agency of Canada. Ottawa (ON): Public Health Agency of Canada; 2012. Available from: http://www.phac-aspc.gc.ca/cd-mc /hale-evas-eng.php

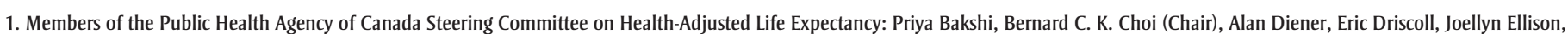
XiaoHong Jiang, Albert Kwan, Lidia Loukine, Wei Luo, Howard Morrison, Heather Orpana, August J. Saaltink, Robert Semenciw, Feng Wang, Chris Waters, Carl Yue, Rita Zhang.

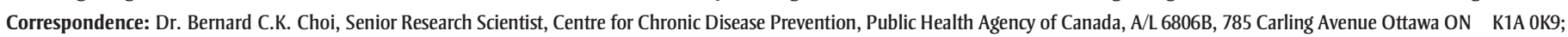
Tel.: 613-957-1074; Email: Bernard.Choi@phac-aspc.gc.ca 